\title{
Fatores relacionados à adesão vacinal em adultos: revisão integrativa
}

\author{
Factors related to immunization adherence in adults: an integrative review \\ Factores relacionados con la adherencia a la inmunización en adultos: una revisión integradora
}

Natália Gomes da Silva ORCID: https://orcid.org/0000-0003-2572-610X Universidade Federal do Triângulo Mineiro, Brasil E-mail: nataliagms16@gmail.com

Lucas Carvalho Santana

ORCID: https://orcid.org/0000-0002-7319-8527 Universidade Federal do Triângulo Mineiro, Brasil E-mail: lucas_enfer@hotmail.com

Lúcia Aparecida Ferreira

ORCID: https://orcid.org/0000-0001-6469-5444 Universidade Federal do Triângulo Mineiro, Brasil E-mail: lap2ferreira@yahoo.com.br

Marina Pereira Rezende

ORCID: https://orcid.org/0000-0003-4054-8911 Universidade Federal do Triângulo Mineiro, Brasil E-mail: marina.rezende@uftm.edu.br

Rejane Cussi Assunção Lemos

ORCID: https://orcid.org/0000-0002-4678-9700

Universidade Federal do Triângulo Mineiro, Brasil

E-mail: rcalenf@yahoo.com.br

\begin{abstract}
Resumo
Objetivo: Verificar os principais fatores relacionados à adesão e à hesitação vacinal em adultos da população geral. Métodologia: Trata-se de uma revisão integrativa da literatura realizada no mês de outubro de 2021 nas bases de dados Medical Literature Analysis and Retrievel System Online (MEDLINE) via National Library of Medicine National Institutes of Health (PubMed), Scopus, Literatura Latino-Americana e do Caribe em Ciências da Saúde (LILACS) e Web of Science. A questão norteadora da pesquisa constituiu-se em: "Quais os fatores relacionados à adesão e à hesitação vacinal em adultos da população geral?". Resultados: Foram elegíveis 30 artigos científicos para a revisão, e foram agrupados em três subgrupos conforme os fatores relacionados à adesão e hesitação abordados no artigo: Confiança/ conhecimento em relação a vacinas e/ou sistema de saúde (14 artigos, 45,2\%); Fatores socioeconômicos (10 artigos, 32,2\%); e Outros fatores (07 artigos, 22,6\%). Conclusão: Os dados obtidos podem contribuir para o desenvolvimento de estratégias de educação em saúde pública, a fim aumentar a adesão à imunização individual, favorecendo a proteção coletiva.
\end{abstract}

Palavras-chave: Aceitação pelo paciente de cuidados de saúde; Imunização; Vacinas.

\begin{abstract}
Objective: To verify the main factors related to vaccine adherence and hesitation in adults from the general population. Methodology: This is an integrative literature review carried out in October 2021 in the Medical Literature Analysis and Retrievel System Online (MEDLINE) databases via the National Library of Medicine National Institutes of Health (PubMed), Scopus, Latino- American and Caribbean in Health Sciences (LILACS) and Web of Science. The guiding question of the research was: "What are the factors related to adherence and vaccine hesitation in adults in the general population?". Results: 30 scientific articles were eligible for review, and were grouped into three subgroups according to the factors related to adherence and hesitation addressed in the article: Trust/knowledge in relation to vaccines and/or health system (14 articles, 45.2\%); Socioeconomic factors (10 articles, 32.2\%); and Other factors (07 articles, 22.6\%). Conclusion: The data obtained can contribute to the development of public health education strategies in order to increase adherence to individual immunization, favoring collective protection.
\end{abstract}

Keywords: Patient acceptance of health care; Immunization; Vaccines.

\section{Resumen}

Objetivo: Verificar los principales factores relacionados con la adherencia y vacilación a la vacuna en adultos de la población general. Metodología: Se trata de una revisión integradora de la literatura realizada en octubre de 2021 en las bases de datos del Medical Literature Analysis and Retrievel System Online (MEDLINE) a través de la Biblioteca Nacional de Medicina de los Institutos Nacionales de Salud (PubMed), Scopus, Latino-American and Caribbean in 
Health Sciences. (LILACS) y Web of Science. La pregunta orientadora de la investigación fue: "¿Cuáles son los factores relacionados con la adherencia y la vacilación a la vacuna en adultos de la población general?". Resultados: Fueron elegibles para revisión 30 artículos científicos, agrupados en tres subgrupos según los factores relacionados con la adherencia y la vacilación abordados en el artículo: Confianza / conocimiento en relación a vacunas y / o sistema de salud (14 artículos, 45,2\%); Factores socioeconómicos (10 artículos, 32,2\%); y Otros factores (07 artículos, 22,6\%). Conclusión: Los datos obtenidos pueden contribuir al desarrollo de estrategias de educación en salud pública con el fin de incrementar la adherencia a la inmunización individual, favoreciendo la protección colectiva.

Palabras clave: Aceptación de la atención de salud; Inmunización; Vacunas.

\section{Introdução}

O processo de imunização é uma das intervenções de saúde mais seguras e econômicas, além de propiciar tanto a imunidade coletiva, quanto a individual (Sacramento, 2021). Favorece o acentuado decréscimo da morbidade e da mortalidade causada pelas doenças imunopreveníveis, devendo ser entendida como um modificador no curso das doenças. A vacinação representa o procedimento de menor custo e maior efetividade, que garante a promoção e a proteção da saúde em indivíduos vacinados (Martins et al., 2018).

A rede pública de saúde brasileira disponibiliza, atualmente, cerca de 300 milhões de doses de imunobiológicos ao ano para combater mais de 19 doenças, em diversas faixas etárias. Dentro do Programa Nacional de Imunizações existe uma série de competências com o objetivo de implementar e implantar as ações relacionadas com a vacinação de caráter obrigatório, estabelecendo critérios do Programa a cargo das secretarias de saúde das unidades federadas (Sacramento, 2021).

Para o estabelecimento do sucesso do processo de imunização é necessário que se tenha boas taxas de cobertura de vacinação e adesão vacinal da população. Considera-se adesão vacinal o fato de iniciar a vacinação e completar o esquema proposto, considerando o número de doses recomendadas e o intervalo entre as mesmas. (Silva, Oliveira \& Galato, 2019).

Apesar da oferta mundial de vacinas e da autorização de uso por órgãos reguladores, indivíduos adeptos ao movimento antivacina questionam a segurança dos imunobiológicos e seus possíveis efeitos colaterais (Corrêa et al., 2021). Esse movimento ganhou força no início de 1998 após a publicação de um artigo na revista The Lancet, na qual uma equipe de médicos afirmou a relação entre o autismo e a vacina tríplice viral. Entretanto, o General Medical Council se posicionou e publicou uma declaração apontando a atitude antiética e irresponsável dos autores que fizeram a publicação. Com isso, o artigo foi retirado dos arquivos do periódico, o autor foi criminalmente responsabilizado e teve o registro médico cassado. (Costa et al., 2020).

Portanto, os movimentos de defesa a liberdade individual e direito a escolher se vacinar ou não, são manifestações contemporâneas. Apesar dos bons resultados alcançados pelos programas de vacinação, a recusa vacinal está presente desde o surgimento da primeira vacina, sendo considerada um dos extremos da hesitação vacinal. (Lim, 2016).

Entende-se por hesitação vacinal o atraso em aceitar ou a recusa das vacinas recomendadas, apesar de sua disponibilidade nos serviços de saúde (MacDonald \& SAGE). De acordo com Sato (2018), esse fenômeno comportamental é bastante complexo em relação a seus determinantes (que envolvem aspectos culturais, sociais e econômicos), e varia ao longo do tempo, do local e dos tipos de vacinas.

Diante disso, o objetivo deste artigo é verificar os principais fatores relacionados à adesão e à hesitação vacinal em adultos da população geral.

\section{Metodologia}

Trata-se de uma revisão integrativa da literatura. Método que sintetiza, de modo ordenado e sistemático, as pesquisas disponíveis sobre determinada temática e direciona a prática fundamentando-se em conhecimento científico (Souza; Silva \& Carvalho, 2010).

A elaboração desta revisão seguiu as seis etapas do método preconizadas por Mendes, Silveira e Galvão (2008), a saber: 
identificação do tema e seleção da hipótese ou questão de pesquisa para a elaboração da revisão integrativa; estabelecimento de critérios para inclusão e exclusão de estudos/ amostragem ou busca na literatura; definição das informações a serem extraídas dos estudos selecionados/ categorização dos estudos; avaliação dos estudos incluídos na revisão integrativa; interpretação dos resultados; e apresentação da revisão/síntese do conhecimento.

Adotou-se a estratégia PICO para a confecção da questão de pesquisa, pois fornece uma estrutura eficiente para a busca de dados em bases eletrônicas. Trata-se de um acrônimo em que o P representa a população a ser estudada; I a intervenção ou exposição a ser investigada; C o controle ou comparação; e O (outcome) o desfecho esperado. (Santos; Pimenta \& Nobre, 2007).

Dessa forma, atribuiu-se ao $\mathrm{P}$ adultos da população geral; ao I os fatores relacionados; o C não foi descrito por não se tratar de um estudo comparativo; e ao O, a adesão e hesitação vacinal. Assim, a questão norteadora da pesquisa constituiu-se em: "Quais os fatores relacionados à adesão e à hesitação vacinal em adultos da população geral?".

O levantamento de dados foi realizado no mês de outubro de 2021 nas bases de dados Medical Literature Analysis and Retrievel System Online (MEDLINE) via National Library of Medicine National Institutes of Health (PubMed), Scopus, Literatura Latino-Americana e do Caribe em Ciências da Saúde (LILACS) e Web of Science. Para a realização das buscas, foram utilizados os descritores em saúde (idioma português) e os /Mesh terms (idioma inglês) "Imunização/ Immunization", "Aceitação pelo Paciente de Cuidados de Saúde/ Patient acceptance of health care" e "Vacinas/ Vaccines", bem como seus sinônimos, combinados entre si com os operadores booleanos OR e AND.

Estabeleceu-se como critérios de inclusão no estudo: artigos resultantes de estudos primários; disponíveis na íntegra em meio eletrônico; que se relacionavam à temática do estudo; publicados em inglês, português e espanhol entre os anos de 2011 e 2021; e realizados em adultos da população geral. Foram excluídos da análise: artigos duplicados, de opinião, de revisão, editoriais, cartas, comentários, notas, teses, dissertações, capítulos de livros e manuais.

O processo de seleção dos artigos foi realizado por dois revisores independentes e com auxílio do software Zotero®.

Para a análise e posterior síntese dos artigos foi utilizado um quadro sinóptico, que destacou os seguintes aspectos: título do artigo; autores; ano; país; método do estudo; principais resultados e conclusões; e nível de evidência do estudo. O nível de evidência foi avaliado conforme a hierarquia utilizada por Fineout-Overholt et al. (2010).

Foram respeitados os aspectos éticos em pesquisa, uma vez que se utilizou artigos científicos disponíveis nas bases de dados e os autores foram devidamente referenciados.

\section{Resultados}

A partir da busca sistemática realizada nas bases de dados, foram encontrados 4094 artigos, sendo 389 artigos na base Web of Science, 10 na LILACS, 1679 na Scopus e 2016 na MedLine. Após a exclusão dos artigos duplicados, restaram 2597 para avaliação do título e resumo. Com a leitura do título e resumo, 2479 artigos foram excluídos por não se adequarem à temática ou à população do estudo.

Com isso, 118 artigos foram selecionados para a leitura na íntegra e nova análise de inclusão no estudo. Assim, foram incluídos 30 artigos nesta revisão, conforme descrito na Figura 1, recomendações do Preferred Reporting Items for Systematic Reviews and Meta-Analyses (PRISMA) (Page et al., 2021). 
Figura 1 - Diagrama PRISMA referente às etapas de busca e seleção dos estudos da revisão integrativa.

\section{Identificação de estudos por meio de bancos de dados}

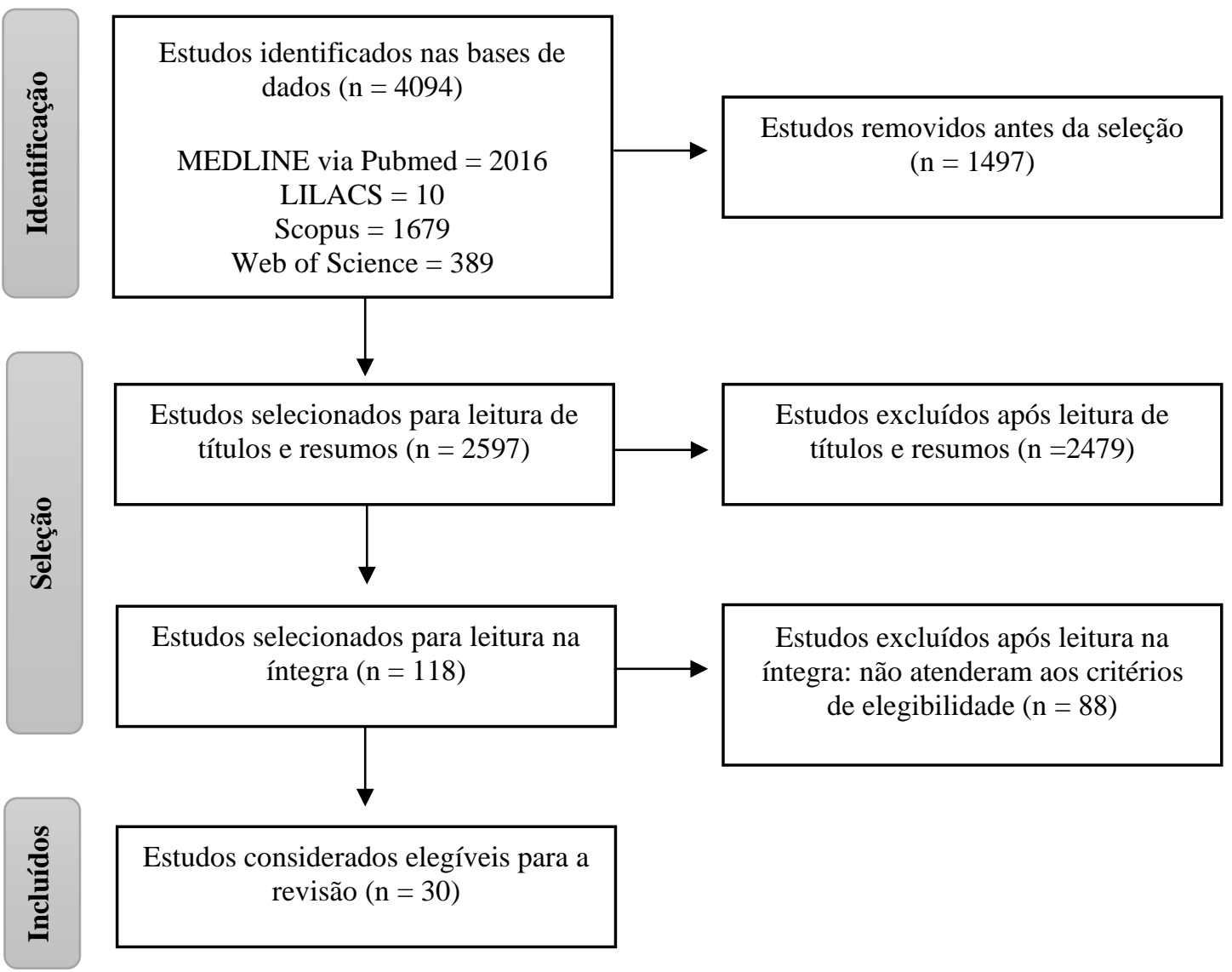

Fonte: Autores (2021)

A síntese dos dados dos artigos elegíveis para a revisão encontra-se no Quadro 1. Os 30 artigos foram agrupados em três subgrupos conforme os fatores relacionados à adesão e à hesitação abordados no artigo: Confiança/ conhecimento em relação a vacinas e/ou sistema de saúde (14 artigos, 45,2\%); Fatores socioeconômicos (10 artigos, 32,2\%); e Outros fatores (7 artigos, 22,6\%). A maioria dos estudos selecionados para a revisão são de delineamento transversal (93,3\%), sendo 70\% (21) com abordagem quantitativa e 30\% (9) qualitativa. Todos os estudos apresentaram nível de evidência VI. 
Quadro 1 - Síntese dos artigos da revisão integrativa.

\begin{tabular}{|c|c|c|}
\hline Título do artigo/ Autores/ Ano/ Localidade & $\begin{array}{l}\text { Método do } \\
\text { estudo }\end{array}$ & Principais resultados/Conclusões \\
\hline $\begin{array}{l}\text { Covid-19 vaccine acceptability and its determinants in } \\
\text { Mozambique: an online survey } \\
\text { Dula } \text { et al., } 2021 \\
\text { Moçambique }\end{array}$ & $\begin{array}{l}\text { Estudo } \\
\text { transversal } \\
\text { realizado de } \\
\text { forma on-line. }\end{array}$ & $\begin{array}{l}\text { Participaram } 1878 \text { pessoas. A aceitação geral da vacina foi de } \\
71,4 \% \text {. os determinantes para a aceitabilidade da vacina foram: } \\
\text { Idade mais avançada, teste covid-19 passado, preocupação de se } \\
\text { tornar (re)infectado pelo COVID-19, ter uma doença crônica e } \\
\text { considerar a vacinação importante para a saúde pessoal e } \\
\text { comunitária. }\end{array}$ \\
\hline $\begin{array}{l}\text { Acceptance and attitudes toward COVID-19 vaccines: a } \\
\text { cross-sectional study from Jordan } \\
\text { El-Elimat et al., } 2021 \\
\text { Jordânia }\end{array}$ & $\begin{array}{l}\text { Transversal e } \\
\text { on-line }\end{array}$ & $\begin{array}{l}3100 \text { participantes. A aceitação pública foi de } 37,4 \% \text {. Os homens, } \\
\text { os que tomaram a vacina contra gripe sazonal, os que acreditavam } \\
\text { na segurança das vacinas, e aqueles que estavam dispostos a pagar } \\
\text { pelas vacinas, quando disponíveis, foram mais propensos a aceitar } \\
\text { vacinas COVID-19 }\end{array}$ \\
\hline $\begin{array}{l}\text { Factors Associated With US Adults' Likelihood of } \\
\text { Accepting COVID-19 Vaccination } \\
\text { Kreps et al., } 2020 \\
\text { Estados Unidos da América }\end{array}$ & Transversal & $\begin{array}{l}\text { Participaram } 1971 \text { adultos. Atributos relacionados à vacina e } \\
\text { características políticas estiveram associados às preferências } \\
\text { autorrelatadas para a escolha de uma vacina hipotética contra a } \\
\text { COVID-19 e vontade autorreferida de receber a vacinação. A } \\
\text { aceitação à vacina hipotética foi maior quando a vacina apresente } \\
\text { uma maior eficácia, um maior tempo de duração da proteção, menor } \\
\text { incidência de efeitos adversos e aprovação definita da Food and } \\
\text { Drug Administration (FDA). }\end{array}$ \\
\hline $\begin{array}{l}\text { Understanding COVID-19 vaccine demand and } \\
\text { hesitancy: A nationwide online survey in China } \\
\text { Lin } \text { et al., } 2020 \\
\text { China }\end{array}$ & $\begin{array}{l}\text { Transversal. On- } \\
\text { line e } \\
\text { autoadministrada }\end{array}$ & $\begin{array}{l}\text { Foram recebidas } 3.541 \text { respostas. A maioria relatou uma intenção } \\
\text { em se vacinar. A percepção de que a vacinação diminui as chances } \\
\text { de obter COVID-19 e não se preocupar com a eficácia de novas } \\
\text { vacinas COVID-19 foram encontrados com as maiores chances } \\
\text { significativas de uma intenção definitiva de tomar a vacina COVID- } \\
\text { 19. A maioria estava confiante na vacina COVID-19 fabricada no } \\
\text { mercado interno, porém } 64,2 \% \text { relataram preferência por uma } \\
\text { vacina COVID-19 fabricada no exterior. }\end{array}$ \\
\hline $\begin{array}{l}\text { Factors likely to affect community acceptance of a } \\
\text { malaria vaccine in two districts of Ghana: A qualitative } \\
\text { study } \\
\text { Meñaca et al., } 2014 \\
\text { Gana }\end{array}$ & Transversal & $\begin{array}{l}\text { Realizado com } 107 \text { pessoas. As comunidades valorizaram muito as } \\
\text { vacinas e citaram a vacinação como a principal motivação para levar } \\
\text { as crianças ao serviço de saúde. No entanto, o conhecimento das } \\
\text { vacinas específicas e o que elas fazem foi limitado. Relatam que } \\
\text { efeitos adversos graves poderiam impedi-los de se vacinarem. }\end{array}$ \\
\hline $\begin{array}{l}\text { Exploring communication, trust in government, and } \\
\text { vaccination intention later in the } 2009 \mathrm{H} 1 \mathrm{~N} 1 \text { pandemic: } \\
\text { results of a national survey } \\
\text { Quinn } \text { et al., } 2013 \\
\text { Estados Unidos da América }\end{array}$ & $\begin{array}{l}\text { Transversal e } \\
\text { on-line }\end{array}$ & $\begin{array}{l}2079 \text { participantes. A comunicação clara e consistente por parte dos } \\
\text { porta-vozes da saúde pública e do governo sobre a vacinação durante } \\
\text { a pandemia H1N1 de } 2009 \text { foi importante para a confiança do } \\
\text { público e aumentou a probabilidade de as pessoas buscarem a } \\
\text { vacina. Outros fatores que contribuíram para a aceitação da } \\
\text { vacinação foram a qualidade da comunicação, acompanhando de } \\
\text { perto as notícias, e a confiança na vacina por causa de um efeito } \\
\text { modelo quando o presidente Obama teve suas filhas imunizadas. }\end{array}$ \\
\hline $\begin{array}{l}\text { Factors influencing SARS-Cov-2 vaccine acceptance } \\
\text { and hesitancy in a population-based sample in Italy } \\
\text { Riccio et al., } 2021 \\
\text { Itália }\end{array}$ & $\begin{array}{l}\text { Transversal e } \\
\text { on-line. }\end{array}$ & $\begin{array}{l}\text { Participaram } 7.605 \text { pessoas. } 81,9 \% \text { da amostra estava inclinada a se } \\
\text { vacinar. Sexo masculino, alto nível de confiança nas instituições de } \\
\text { saúde/ governo e crenças pessoais sobre a segurança das vacinas } \\
\text { foram encontradas entre os preditores significativos da aceitação à } \\
\text { vacinação contra a COVID-19. }\end{array}$ \\
\hline $\begin{array}{l}\text { Prevalence of influenza vaccine hesitancy at a tertiary } \\
\text { care hospital in Riyadh, Saudi Arabia } \\
\text { Alabbad } \text { et al., } 2018 \\
\text { Arábia Saudita }\end{array}$ & Trans & $\begin{array}{l}\text { Dos } 300 \text { participantes do estudo, } 17 \% \text { expressaram hesitação } \\
\text { vacinal. As razões mais comuns dadas para a recusa vacinal foram: } \\
\text { "Não tem nenhum efeito ou benefício positivo" [21\%], "Não preciso } \\
\text { porque sou saudável" [17\%], e "Acho que causa sérios efeitos } \\
\text { colaterais" [13\%]. Não houve relação significativa entre o nível de } \\
\text { escolaridade e o recebimento da vacinação contra a gripe. A } \\
\text { hesitação da vacina contra a gripe foi considerada baixa. A razão } \\
\text { mais comum para a recusa vacinal foi acreditar que ela não teve } \\
\text { efeito positivo e que é desnecessária. }\end{array}$ \\
\hline
\end{tabular}




\begin{tabular}{|c|c|c|}
\hline $\begin{array}{l}\text { The Decision to Vaccinate or not during the H1N1 } \\
\text { Pandemic: Selecting the Lesser of Two Evils? } \\
\text { Ashbaugh et al., } 2013 \\
\text { Canadá }\end{array}$ & $\begin{array}{l}\text { Transversal e } \\
\text { online }\end{array}$ & $\begin{array}{l}817 \text { respostas. A associação mais consistente e mais forte foi que as } \\
\text { crenças negativas sobre a vacina H1N1 (por exemplo, o medo de } \\
\text { seus efeitos colaterais) estavam relacionadas à decisão de não ser } \\
\text { vacinada, enquanto as crenças sobre os perigos do vírus H1N1 } \\
\text { estavam relacionadas à decisão de ser vacinado. Mais notavelmente, } \\
\text { ter crenças negativas muito fortes sobre a vacina era um preditor } \\
\text { mais poderoso do que mesmo crenças fortes sobre os perigos do } \\
\text { vírus H1N1. Além disso, a obtenção de informações da internet, em } \\
\text { comparação com fontes mais tradicionais de informação (por } \\
\text { exemplo, TV, jornais) estava relacionada à decisão de não ser } \\
\text { vacinada. }\end{array}$ \\
\hline $\begin{array}{l}\text { A comparison of the seasonal influenza vaccination } \\
\text { rates and related factors } \\
\text { Başpınar } \text { et al., } 2020 \\
\text { Istambul }\end{array}$ & Transversal & $\begin{array}{l}\text { A taxa de vacinação contra gripe entre } 391 \text { pacientes (idade média: } \\
39,9 \pm 13,5 \text { anos) foi de } 22,5 \% \text { ( } 88 / 391) \text {, e a taxa de participantes que } \\
\text { foram recomendados para receber a vacina foi de } 25,3 \% \text { de todos } \\
\text { com concordância moderada, embora } 81,3 \% \text { dos indivíduos } \\
\text { acreditassem que a vacina era benéfica e metade dos pacientes } \\
(50,5 \%) \text { reagiu positivamente quando seu médico recomendou. } \\
\text { Dezessete pontos nove dos pacientes que apresentavam doenças } \\
\text { semelhantes à gripe no ano anterior }(72,6 \%) \text { haviam sido vacinados. } \\
\text { Efeito colateral experimentado, medo de efeitos colaterais e injeçãa, } \\
\text { e hesitação vacinal não eram barreiras à aceitação da vacinação. A } \\
\text { presença de recomendação; Intervalo de confiança de } 95 \% \text {, } \\
\text { informações sobre o tempo de vacinação e crença sobre benefícios } \\
\text { da vacina foram os preditores da aceitação da vacina. }\end{array}$ \\
\hline $\begin{array}{l}\text { Barriers, facilitators, and potential strategies for } \\
\text { increasing HPV vaccination: A statewide assessment to } \\
\text { inform } \\
\text { action } \\
\text { Cartmell } \text { et al., } 2018 \\
\text { Carolina do Sul }\end{array}$ & Transversal & $\begin{array}{l}34 \text { participantes. As barreiras à vacinação contra o Papilomavírus } \\
\text { Humano (HPV) incluíam falta de conscientização sobre o HPV, falta } \\
\text { de recomendação do provedor, preocupações com vacinas contra o } \\
\text { HPV e falta de acesso. Os facilitadores incluíram o impulso para } \\
\text { melhorar a vacinação contra o HPV, a exigência de vacinação contra } \\
\text { tétano na matrícula escolar, a vacinação contra o HPV na rede de } \\
\text { farmácias, o registro estadual de imunização e o financiamento da } \\
\text { vacina contra o HPV. }\end{array}$ \\
\hline $\begin{array}{l}\text { Patient's behaviors and missed opportunities for } \\
\text { vaccination against seasonal epidemic influenza and } \\
\text { evaluation of their impact on patient's influenza vaccine } \\
\text { uptake. } \\
\text { Casalino et al., } 2018 \\
\text { França }\end{array}$ & Transversal & $\begin{array}{l}868 \text { participantes. A captação vacinal foi de } 33,2 \%, 42 \% \text { dos } \\
\text { pacientes sabiam da possível gravidade da gripe, } 23 \% \text { achavam que } \\
\text { não estavam em risco de influenza grave, } 39 \% \text { sabiam que tinham } \\
\text { indicação para a vacina e } 4,3 \% \text { a } 11,5 \% \text { expressavam reservas sobre } \\
\text { efeitos colaterais intravenosos e eficácia. O "vale-saúde" foi } \\
\text { utilizado por } 44,3 \% \text { dos pacientes, mas apenas } 14,8 \% \text { foram } \\
\text { vacinados. Foram notificados } 484 \text { pacientes }(69,4 \%) \text { declarando } \\
1104 \text { consultas e } 148 \text { vacinações contra gripe }(86,6 \%) \text {. Os preditores } \\
\text { da captação vacinal foram: conhecimento de formas de influenza } \\
\text { graves e fatais; confiança na eficácia da vacina contra a gripe; } \\
\text { oposição às vacinas; visita ao clínico geral; clínico geral proposto. }\end{array}$ \\
\hline $\begin{array}{l}\text { Acceptance of a COVID-19 Vaccine in Southeast Asia: } \\
\text { A Cross-Sectional Study in Indonesia. } \\
\text { Harapan } \text { et al., } 2020 \\
\text { Indonésia }\end{array}$ & $\begin{array}{l}\text { Transversal e } \\
\text { on-line }\end{array}$ & $\begin{array}{l}1.359 \text { entrevistados. A aceitação de uma vacina COVID-19 pela } \\
\text { população geral foi altamente influenciada pela eficácia da linha de } \\
\text { base da vacina. Independente da taxa de eficácia, ser profissional de } \\
\text { saúde esteve associado a maior aceitação. }\end{array}$ \\
\hline $\begin{array}{l}\text { Covid-19 vaccine acceptance in the Democratic } \\
\text { Republic of Congo: A cross-sectional survey } \\
\text { Ditekemena et al., } 2021 \\
\text { República Democrática do Congo }\end{array}$ & $\begin{array}{l}\text { Estudo } \\
\text { transversal } \\
\text { realizado de } \\
\text { forma on-line. }\end{array}$ & $\begin{array}{l}\text { Foram incluídas } 4131 \text { respostas. } 55,9 \% \text { indicaram estar dispostos a } \\
\text { serem vacinados. Apresentou associação à maior disposição para } \\
\text { vacinação: renda média e alta, ser testado para COVID-19 e o } \\
\text { reconhecimento da existência da doença. Ser profissional de saúde } \\
\text { esteve associado à diminuição da aceitação à vacinação. }\end{array}$ \\
\hline $\begin{array}{l}\text { Factors influencing likelihood of COVID-19 } \\
\text { vaccination: A survey of Tennessee adults } \\
\text { Gatwood } \text { et al., } 2021 \\
\text { Estados Unidos da América }\end{array}$ & Transversal & $\begin{array}{l}1000 \text { participantes. } 34,4 \% \text { dos entrevistados indicou alguma } \\
\text { hesitação vacinal histórica, e apenas } 21,4 \% \text { indicaram sempre a } \\
\text { vacinação contra a gripe sazonal. 54,1\%) indicou pelo menos } \\
\text { alguma hesitação em relação à vacinação contra o COVID-19. A } \\
\text { hesitação vacinal foi mais provável entre os negros americanos, as } \\
\text { pessoas com posturas conservadoras e população residente de áreas } \\
\text { não metropolitanas. }\end{array}$ \\
\hline $\begin{array}{l}\text { Knowledge, attitudes and practices about influenza } \\
\mathrm{A}(\mathrm{H} 1 \mathrm{~N} 1) \text { 2009, and influenza vaccine in Mexico: } \\
\text { Results of a population survey. } \\
\text { Jimenez-Corona et al., } 2012 \\
\text { México }\end{array}$ & Transversal & $\begin{array}{l}\text { Foram entrevistados } 1600 \text { adultos. } 34 \% \text { já haviam recebido vacina } \\
\text { contra gripe sazonal e } 90,6 \% \text { estavam dispostos a ser vacinados } \\
\text { contra gripe } \mathrm{A}(\mathrm{H} 1 \mathrm{~N} 1) \text {. A escolaridade e a idade influenciam a } \\
\text { rejeição para a vacinação. }\end{array}$ \\
\hline
\end{tabular}




\begin{tabular}{|c|c|c|}
\hline $\begin{array}{l}\text { COVID-19 Vaccination Hesitancy in the United States: } \\
\text { A Rapid National Assessment } \\
\text { Khubchandani } \text { et al., } 2021 \\
\text { Estados Unidos da América }\end{array}$ & Transversal & $\begin{array}{l}\text { Participaram do estudo } 1878 \text { indivíduos. A probabilidade de obter } \\
\text { uma imunização contra COVID-19 na população do estudo foi: } \\
\text { muito provável (52\%), um pouco provável }(27 \%) \text {, não provável } \\
(15 \%) \text {, definitivamente não (7\%). A hesitação vacinal foi maior } \\
\text { entre afro-americanos (34\%), hispânicos (29\%), aqueles que tiveram } \\
\text { filhos em casa }(25 \%) \text {, moradores rurais }(29 \%) \text {, pessoas no nordeste } \\
\text { dos Estados Unidos }(25 \%) \text { e aqueles que se identificaram como } \\
\text { republicanos }(29 \%) \text {. }\end{array}$ \\
\hline $\begin{array}{l}\text { Determinants of COVID-19 vaccine acceptance in the } \\
\text { US } \\
\text { Malik et al., } 2020 \\
\text { Estados Unidos da América }\end{array}$ & $\begin{array}{l}\text { Transversal e } \\
\text { on-line. }\end{array}$ & $\begin{array}{l}672 \text { participantes. } 67 \% \text { disseram que aceitariam uma vacina } \\
\text { COVID-19 se fosse recomendada para eles. Ao relacionar os fatores } \\
\text { sociodemográficos: homens, idosos e pessoas com nível de } \\
\text { escolaridade superior eram mais propensos a aceitar a vacina. } \\
\text { Apesar de uma boa taxa de aceitação (67\%), houve disparidades } \\
\text { demográficas e geográficas perceptíveis na aceitação da vacina. }\end{array}$ \\
\hline $\begin{array}{l}\text { Understanding and increasing influenza vaccination } \\
\text { acceptance: insights from a } 2016 \text { national survey of U.S. } \\
\text { adults } \\
\text { Nowak et al., } 2018 \\
\text { Estados Unidos da América }\end{array}$ & Transversal & $\begin{array}{l}\text { Participaram } 1005 \text { indivíduos. Os entrevistados hispânicos e aqueles } \\
\text { com menos de } 64 \text { anos eram muito menos propensos a se vacinar } \\
\text { contra a gripe. Também estavam menos conscientes da } \\
\text { recomendação, menos informados sobre a gripe e os benefícios da } \\
\text { vacinação e menos confiantes na vacina. }\end{array}$ \\
\hline $\begin{array}{l}\text { Cross-sectional analysis of COVID-19 vaccine } \\
\text { intention, perceptions and hesitancy across Latin } \\
\text { America and the Caribbean } \\
\text { Urrunaga-Pastor } \text { et al., } 2021 \\
\text { América Latina e Caribe }\end{array}$ & Tran & $\begin{array}{l}\text { Participaram } 472.521 \text { latino-americanos. } 80 \% \text { da amostra } \\
\text { apresentou intenção vacinal contra a COVID-19. Pessoas dos sexos } \\
\text { feminino e não binário estavam associadas a uma menor } \\
\text { probabilidade de aceitação da vacina. Além disso, o medo de se } \\
\text { infectar ou de um familiar se infectar com a forma grave da doença } \\
\text { esteve associado a uma maior probabilidade de aceitação da vacina. }\end{array}$ \\
\hline $\begin{array}{l}\text { Determinants of covid-19 vaccine acceptance in Saudi } \\
\text { Arabia: A web-based national survey } \\
\text { Al-Mohaithef \& Padhi, } 2020 \\
\text { Arábia Saudita }\end{array}$ & Transversal & $\begin{array}{l}\text { Dos } 992 \text { entrevistados, } 642 \text { demonstraram interesse em aceitar a } \\
\text { vacina COVID-19 se ela estiver disponível. A vontade de aceitar a } \\
\text { futura vacina COVID-19 é relativamente alta entre as faixas etárias } \\
\text { mais velhas, sendo participantes casados com pós-graduação em } \\
\text { nível de escolaridade ou superior }(68,8 \%) \text {, não-sauditas }(69,1 \%) \text {, } \\
\text { empregados no setor governamental ( }(68,9 \%) \text {. No modelo } \\
\text { multivariado, os entrevistados com mais de } 45 \text { anos e casados } \\
\text { estiveram significativamente associados à aceitação da vacina. }\end{array}$ \\
\hline $\begin{array}{l}\text { As the pandemic progresses, how does willingness to } \\
\text { vaccinate against COVID-19 evolve? } \\
\text { Alley et al., } 2021 \\
\text { Austrália }\end{array}$ & $\begin{array}{l}\text { Estudo } \\
\text { longitudinal }\end{array}$ & $\begin{array}{l}2343 \text { adultos australianos. A vontade de vacinar foi menor em } \\
\text { pessoas com certificado ou diploma (79\%) em comparação com } \\
\text { aquelas com bacharelado ( } 87 \%) \text { e menor em usuários infrequentes } \\
\text { da mídia tradicional }(78 \%) \text { em comparação com usuários frequentes } \\
\text { da mídia tradicional }(89 \%) \text {. As mulheres eram mais propensas a não } \\
\text { ter certeza se estariam dispostas a vacinar (10\%) em relação aos } \\
\text { homens ( } 7 \% \text { ). Não houve associação entre vontade de vacinar e } \\
\text { idade, doença crônica ou uso de redes sociais. }\end{array}$ \\
\hline $\begin{array}{l}\text { COVID-19 vaccine hesitancy and resistance: Correlates } \\
\text { in a nationally representative longitudinal survey of the } \\
\text { Australian population } \\
\text { Edwards } \text { et al., } 2021 \\
\text { Austrália }\end{array}$ & $\begin{array}{l}\text { longitudinal } \\
\text { realizado de } \\
\text { forma on-line. }\end{array}$ & $\begin{array}{l}3.061 \text { entrevistados. } 59 \% \text { receberiam definitivamente a vacina, } 29 \% \\
\text { tinham baixos níveis de hesitação, } 7 \% \text { tinham altos níveis de } \\
\text { hesitação e } 6 \% \text { eram resistentes. Aqueles que tinham níveis mais } \\
\text { elevados de renda familiar, níveis mais elevados de distanciamento } \\
\text { social, que tinham mais confiança em seu governo de estado ou } \\
\text { território ou confiança em seus hospitais, ou apoiavam mais a } \\
\text { migração eram mais propensos a se vacinar. }\end{array}$ \\
\hline $\begin{array}{l}\text { Vaccine hesitancy: the next challenge in the fight } \\
\text { against COVID-19 } \\
\text { Dror } \text { et al., } 2020 \\
\text { Israel }\end{array}$ & Transversal & $\begin{array}{l}\text { Foram preenchidos } 1941 \text { questionários. Profissionais de saúde } \\
\text { envolvidos no cuidado de pacientes com COVID-19, e indivíduos } \\
\text { que se consideravam em risco da doença, eram mais propensos à } \\
\text { adesão à vacinação COVID-19 se e quando disponíveis. Em } \\
\text { contrapartida, pessoas que não estavam em contato direto com } \\
\text { pessoas positivas de COVID-19 expressaram níveis mais elevados } \\
\text { de hesitação vacinal. }\end{array}$ \\
\hline
\end{tabular}




\begin{tabular}{|c|c|c|}
\hline $\begin{array}{l}\text { Acceptance of a COVID-19 vaccine and its related } \\
\text { determinants among the general adult population in } \\
\text { Kuwait } \\
\text { Alqudeimat } \text { et al., } 2021 \\
\text { Kuwait }\end{array}$ & $\begin{array}{l}\text { Transversal e } \\
\text { online }\end{array}$ & $\begin{array}{l}2.368 \text { participantes. No total, } 53,1 \% \text { dos participantes estavam } \\
\text { dispostos a aceitar uma vacina COVID-19 uma vez disponível. Os } \\
\text { homens estavam mais dispostos a aceitar uma vacina COVID-19 do } \\
\text { que as mulheres (58,3 vs. 50,9\%). Os indivíduos que viam as vacinas } \\
\text { em geral com riscos relacionados à saúde estavam menos dispostos } \\
\text { a aceitar a vacinação. Além disso, os participantes que receberam } \\
\text { anteriormente uma vacina contra a gripe tiveram maior } \\
\text { probabilidade de aceitar uma vacina COVID- } 19 \text {. A vontade de se } \\
\text { vacinar contra o COVID-19 aumentou à medida que as chances de } \\
\text { contrair a infecção aumentaram. No total, } 53,1 \% \text { dos participantes } \\
\text { do estudo demonstraram vontade de se vacinar contra o COVID-19. }\end{array}$ \\
\hline $\begin{array}{l}\text { Reasons for low influenza vaccination coverage among } \\
\text { adults in Puerto Rico, influenza season 2013-2014 } \\
\text { Arriola } \text { et al., } 2015 \\
\text { Porto Rico }\end{array}$ & $\begin{array}{l}\text { Transversal. } \\
\text { Pesquisa } \\
\text { telefônica de } \\
\text { discagem } \\
\text { aleatória }\end{array}$ & $\begin{array}{l}\text { Dos } 439 \text { pesquisados, } 229 \text { concluíram a pesquisa com uma taxa de } \\
\text { resposta de } 52 \% \text {. A idade mediana dos entrevistados foi de } 55 \text { anos; } \\
18 \% \text { referiam ter recebido vacinação contra a gripe } 2013-2014 \text {. } \\
\text { Entre os } 180 \text { entrevistados não vacinados, } 38 \% \text { relataram barreiras } \\
\text { associadas ao acesso limitado à vacinação, } 24 \% \text { relataram não } \\
\text { querer ou precisar de vacinação contra a gripe e } 20 \% \text { relataram } \\
\text { preocupaçães com a segurança. As taxas de vacinação foram mais } \\
\text { elevadas entre os adultos que receberam recomendação e/ou oferta } \\
\text { de vacinação contra gripe (43\% vs. } 14 \% \text { ) }\end{array}$ \\
\hline $\begin{array}{l}\begin{array}{l}\text { Converting the maybes: Crucial for a } \\
\text { vavin-19 }\end{array} \\
\text { Attwell } \text { et al., } 2021 \\
\text { Austrália }\end{array}$ & $\begin{array}{l}\text { Transversal } \\
\text { online }\end{array}$ & $\begin{array}{l}1.313 \text { respostas. } 65 \% \text { estavam dispostos a vacinar, sendo } 27 \% \text { na } \\
\text { categoria "talvez". Os entrevistados eram mais propensos a estar no } \\
\text { grupo "talvez" do que o "sim" quando perceberam que o COVID-19 } \\
\text { era menos grave, tinham menos confiança na ciência, estavam } \\
\text { menos dispostos a vacinar para a gripe e eram do sexo feminino. } \\
\text { Eles eram mais propensos a estar no grupo "talvez" do que "não" } \\
\text { quando eles perceberam o COVID- } 19 \text { como grave, e menos } \\
\text { propensos a ser uma farsa, tinham mais confiança na ciência e maior } \\
\text { disposição para vacinar a gripe. Uma repetição da pesquisa em } \\
\text { novembro de } 2020 \text { com um subconjunto de participantes encontrou } \\
\text { menor número de participantes dizendo "sim" à vacina }(56 \%) \text { e } \\
\text { mais participantes dizendo "talvez" (31\%). }\end{array}$ \\
\hline $\begin{array}{l}\text { COVID-19 vaccine hesitancy and related fears and } \\
\text { anxiety } \\
\text { Bendau et al., } 2021 \\
\text { Alemanha }\end{array}$ & $\begin{array}{l}\text { Transversal e } \\
\text { online }\end{array}$ & $\begin{array}{l}1.779 \text { participantes. } 64,5 \% \text { da amostra afirmaram que aceitariam } \\
\text { absolutamente a vacinação, } 13,8 \% \text { preferiram aceitá-la, } 10,4 \% \\
\text { estavam indecisas e } 5,2 \% \text { preferiram não e } 6,0 \% \text { absolutamente não } \\
\text { se vacinariam. A ansiedade relacionada ao COVID-19 e os temores } \\
\text { de infecção e consequências relacionadas à saúde correlacionam-se } \\
\text { significativamente de forma positiva em relação a aceitação da } \\
\text { vacina. Em contrapartida, os temores sociais e econômicos } \\
\text { apresentaram associações negativas significativas com a disposição } \\
\text { da vacinação. Os construtos mais amplos de ansiedade inespecífica } \\
\text { e sintomas depressivos não foram significativamente associados à } \\
\text { aceitação da vacina. A aceitação da vacina difere entre usuários/não } \\
\text { usuários de redes sociais e sites oficiais para obter informações sobre } \\
\text { a pandemia. A ansiedade relacionada ao COVID-19 es medos } \\
\text { relacionados à saúde estiveram associados à maior aceitação da } \\
\text { vacina, enquanto o medo das consequências sociais e econômicas } \\
\text { mostrou a direção contrária. }\end{array}$ \\
\hline $\begin{array}{l}\begin{array}{l}\text { Determinants of influenza vaccination among a large } \\
\text { adult population }\end{array} \\
\text { Baron } \text { et al., } 2018 \\
\text { Canadá }\end{array}$ & Trai & $\begin{array}{l}4.620 \text { entrevistas. Entre os grupos-alvo, } 55,4 \% \text { dos adultos com } \\
\text { idade } \geq 60 \text { e } 32,2 \% \text { dos adultos de } 18 \text { a } 59 \text { anos com pelo menos uma } \\
\text { doença crônica receberam a vacina contra a gripe durante a } \\
\text { temporada 2013-2014. Vários determinantes foram } \\
\text { significativamente associados à vacinação contra a gripe em ambos } \\
\text { os grupos, como ter recebido recomendação de um profissional de } \\
\text { saúde. Entre os adultos com } \geq 60 \text { anos, não ter consultado } \\
\text { quiroprático nos últimos } 12 \text { meses, não fumantes e autopercepção } \\
\text { do estado de saúde ruim estiveram significativamente ligados à } \\
\text { vacinação contra a gripe. No grupo mais jovem, a vacinação contra } \\
\text { a gripe esteve independentemente associada ao baixo consumo de } \\
\text { álcool e ao excesso de peso. }\end{array}$ \\
\hline $\begin{array}{l}\text { A global survey of potential acceptance of a COVID-19 } \\
\text { vaccine } \\
\text { Lazarus et al., } 2021 \\
\text { Multinacional, em } 19 \text { países. }\end{array}$ & Transversal & $\begin{array}{l}13.426 \text { pessoas. } 71,5 \% \text { dos participantes relataram que teriam } \\
\text { intenção de tomar uma vacina contra a COVID-19. Os entrevistados } \\
\text { que relataram níveis mais altos de confiança nas informações de } \\
\text { fontes governamentais eram mais propensos a aceitar uma vacina e } \\
\text { tomar o conselho de seu empregador para fazê-lo. }\end{array}$ \\
\hline
\end{tabular}

Fonte: Autores (2021). 


\section{Discussão}

Diversos estudos foram realizados a fim de verificar a aceitação de uma possível vacina contra a COVID-19, no período pandêmico (Alley et al, 2021; Alqudeimat et al, 2021; Bendau et al, 2021; Ditekemena et al., 2021; Dula et al., 2021; Edwards et al., 2021; El-Elimat et al., 2021; Gatwood et al., 2021; Khubchandani et al., 2021; Lazarus et al., 2021; Lin et al., 2020; Riccio et al, 2021; Urrunaga-Pastor et al, 2021; Al-Mohaithef \& Padhi, 2020; Dror et al., 2020; Harapan et al., 2020; Kreps et al., 2020; Malik et al, 2020). Nestes estudos, foi demonstrado que a população teoricamente mais exposta ao risco de adoecer, está mais suscetível à aceitação da vacina em relação às demais parcelas da população. Dado corroborado por um estudo realizado no México por Jiménez-Corona et al. (2009), que evidencia que a percepção de risco da doença está diretamente ligada ao comportamento em relação à saúde, sendo reconhecido como um fator chave que modifica a atitude no momento da aceitação vacinal. Assim, uma pessoa que se sente em grande risco de adoecer buscará proteção e terá atitudes mais positivas em relação à vacinação em geral.

É provável que o manejo inadequado das informações impacte negativamente parte da população, o que gera desconfiança e dúvidas sobre a existência da doença e sua gravidade, bem como sobre a eficácia da vacina. O primeiro estudo dos EUA a explorar empiricamente a qualidade da comunicação e confiança nas ações governamentais durante uma pandemia, mostrou que funcionários de saúde pública eram os porta-vozes de maior confiança, seguido por um ex-presidente do país, que optou por vacinar suas filhas. Vários estudos enfatizaram a importância da honestidade, abertura e transparência no que diz respeito à tomada de decisões e confiança na comunicação em uma pandemia. (Quinn et al, 2013).

Esses dados evidenciam a necessidade de fortalecer campanhas e informar adequadamente a população sobre os riscos das doenças, bem como adequar as informações ao nível de entendimento de toda a população. Segundo Lin et al. (2020), as sugestões externas às campanhas vacinais são consideradas importantes, ou seja, o fornecimento de informações abrangentes e adequadas ao público, com linguagem acessível à todas as classes, baseadas em fortes evidências de segurança e eficácia da vacina constatadas em pesquisas de campo são diferenciais importantes em relação à adesão vacinal.

Um estudo realizado no Kuwait identificou vários fatores associados à aceitação de uma vacina contra COVID-19, homens eram mais prováveis do que as mulheres para aceitar a vacinação, o que está de acordo com achados anteriores. Além disso, a aceitabilidade foi maior entre os indivíduos com 21-24 anos (74,3\%) e menor entre aqueles com idade entre 55-64 anos $(35,3 \%)$ (Alqudeimat, 2020).

Essas observações são semelhantes às descobertas de um estudo realizado entre adultos nos Estados Unidos, que mostrou que indivíduos com idades entre 18-29 anos tiveram maior aceitabilidade (71\%) do que aqueles com idade entre 50-64 anos (64\%) (Reiter et al., 2020). Em contradição, outros estudos mostraram que a aceitabilidade aumenta com a idade. Esses achados conflitantes podem ser explicados por diferenças regionais nas populações, nas percepções e crenças sobre a vacinação, que difere entre grupos de idade. (Malik et al., 2020).

Além disso, foi observado que grupos familiares com renda mais elevada (Ditekemena et al., 2021; Edwards et al., 2021) bem como pessoas com nível de escolaridade superior eram mais propensos a aceitar a vacina (Al-Mohaithef \& Padhi, 2020; Malik et al, 2020). Tal ocorrência pode ser explicada pelo fato de as pessoas com maior nível de escolaridade terem mais facilidade de entendimento em relação à importância da vacinação, além dos riscos inerentes à não-vacinação. Outro ponto a ser discutido é o acesso à vacinação, que pode estar facilitado para as pessoas com renda mais elevada.

Já em relação à hesitação vacinal, percebeu-se que foi mais provável entre os negros americanos, as pessoas com posturas conservadoras e população residente em áreas não metropolitanas (Gatwood et al., 2021; Khubchandani et al., 2021). Dados que são endossados por estudo realizado por Doherty et al. (2021) na Carolina do Norte. Segundo os autores, a hesitação vacinal 
Research, Society and Development, v. 11, n. 1, e0911124194, 2022

(CC BY 4.0) | ISSN 2525-3409 | DOI: http://dx.doi.org/10.33448/rsd-v11i1.24194

tende a persistir entre populações historicamente marginalizadas, a menos que recursos substanciais, financiamento, atividades de saúde pública e acesso a programas de vacinação sejam implantados nessas populações.

\section{Conclusão}

Os principais fatores de interferência na decisão vacinal estão relacionados à confiança/conhecimento em relação a vacinas e/ou sistema de saúde e a fatores socioeconômicos. Outros fatores como confiança no governo, medo da doença e confiança na própria saúde também foram encontrados, no entanto, em menor prevalência.

Esses resultados podem contribuir no desenvolvimento de estratégias de educação em saúde pública, a fim aumentar a adesão à imunização individual, favorecendo a proteção coletiva.

Apesar de a hesitação vacinal ser um problema conhecido, apontar seus fatores relacionados ainda é um desafio, já que seus determinantes variam ao longo do tempo e são específicos de cada contexto e região.

Como limitação do estudo, pode-se citar o fato de terem sido excluídos os artigos que não estavam disponíveis gratuitamente nas bases de dados. Sugere-se a realização de novos estudos acerca da temática, a fim de verificar a adesão e hesitação vacinal da população brasileira.

\section{Referências}

Alabbad, A. A., Alsaad, A. K., Al Shaalan, M. A., Alola, S., \& Albanyan, E. A. (2018). Prevalence of influenza vaccine hesitancy at a tertiary care hospital in Riyadh, Saudi Arabia. Journal of Infection and Public Health, 11(4), 491-499. Scopus. https://doi.org/10.1016/j.jiph.2017.09.002

Alley, S. J., Stanton, R., Browne, M., To, Q. G., Khalesi, S., Williams, S. L., Thwaite, T. L., Fenning, A. S., \& Vandelanotte, C. (2021). As the pandemic progresses, how does willingness to vaccinate against covid-19 evolve? International Journal of Environmental Research and Public Health, 18(2), 1-14. Scopus. https://doi.org/10.3390/ijerph18020797

Al-Mohaithef, M., \& Padhi, B. K. (2020). Determinants of covid-19 vaccine acceptance in saudi arabia: A web-based national survey. Journal of Multidisciplinary Healthcare, 13, 1657-1663. Scopus. https://doi.org/10.2147/JMDH.S276771

Alqudeimat, Y., Alenezi, D., Alhajri, B., Alfouzan, H., Almokhaizeem, Z., Altamimi, S., Almansouri, W., Alzalzalah, S., \& Ziyab, A. H. (2021). Acceptance of a COVID-19 vaccine and its related determinants among the general adult population in Kuwait. Medical Principles and Practice, 30(3), 262-271. Scopus. https://doi.org/10.1159/000514636

Arriola, C. S., Mercado-Crespo, M. C., Rivera, B., Serrano-Rodriguez, R., Macklin, N., Rivera, A., Graitcer, S., Lacen, M., Bridges, C. B., \& Kennedy, E. D. (2015). Reasons for low influenza vaccination coverage among adults in Puerto Rico, influenza season 2013-2014. Vaccine, 33(32), 3829-3835. Scopus. https://doi.org/10.1016/j.vaccine.2015.06.093

Ashbaugh, A. R., Herbert, C. F., Saimon, E., Azoulay, N., Olivera-Figueroa, L., \& Brunet, A. (2013). The Decision to Vaccinate or Not during the H1N1 Pandemic: Selecting the Lesser of Two Evils? PLoS ONE, 8(3), Article 3. Scopus. https://doi.org/10.1371/journal.pone.0058852

Attwell, K., Lake, J., Sneddon, J., Gerrans, P., Blyth, C., \& Lee, J. (2021). Converting the maybes: Crucial for a successful COVID-19 vaccination strategy. PLoS ONE, 16(1 January), Article 1 January. Scopus. https://doi.org/10.1371/journal.pone.0245907

Baron, G., Gosselin, V., Petit, G., Guay, M., \& Gagneur, A. (2018). Determinants of influenza vaccination among a large adult population in Quebec. Human Vaccines and Immunotherapeutics, 14(11), 2722-2727. Scopus. https://doi.org/10.1080/21645515.2018.1486155

Başpınar, M. M., Tanımlı, E., Depreli, S. G., Aktaş, H. K., Aktaş, R., \& Basat, O. (2020). A comparison of the seasonal influenza vaccination rates and related factors. Haseki Tip Bulteni, 58(3), 251-258. Scopus. https://doi.org/10.4274/haseki.galenos.2020.5979

Bendau, A., Plag, J., Petzold, M. B., \& Ströhle, A. (2021). COVID-19 vaccine hesitancy and related fears and anxiety. International Immunopharmacology, 97. Scopus. https://doi.org/10.1016/j.intimp.2021.107724

Cartmell, K. B., Young-Pierce, J., McGue, S., Alberg, A. J., Luque, J. S., Zubizarreta, M., \& Brandt, H. M. (2018). Barriers, facilitators, and potential strategies for increasing HPV vaccination: A statewide assessment to inform action. Papillomavirus Research, 5, 21-31. Scopus. https://doi.org/10.1016/j.pvr.2017.11.003

Casalino, E., Ghazali, A., Bouzid, D., Antoniol, S., Pereira, L., Kenway, P., Choquet, C., Bernard, J., Casaubieilh, A., Debit, A., Dupeyrat, E., Gauthier-Gentès, P., Gil, Y., Gillo, M., Goncalves, S., Laurent, N., Macaux, M., Pigeau, J., Ranaivoson, M., ... Roux, L. (2018). Patient's behaviors and missed opportunities for vaccination against seasonal epidemic influenza and evaluation of their impact on patient's influenza vaccine uptake. PLoS ONE, 13(3), Article 3. Scopus. https://doi.org/10.1371/journal.pone.0193029

CorrêaS. M. C., de VasconcelosP. F., dos PassosJ. S., MarquesV. G., TanajuraN. P. M., do NascimentoD. R., SilvaC. S., MagalhãesL. P., NevesL. R., \& SilveiraM. B. S. (2021). As possíveis causas da não adesão à imunização no Brasil: uma revisão de literatura. Revista Eletrônica Acervo Saúde, 13(4), e7030. https://doi.org/10.25248/reas.e7030.2021 
Research, Society and Development, v. 11, n. 1, e0911124194, 2022 (CC BY 4.0) | ISSN 2525-3409 | DOI: http://dx.doi.org/10.33448/rsd-v11i1.24194

Costa, B. B., Viegas, D. de J., Moreira, T. A., \& Abreu, P. A. (2020). O movimento antivacina no YouTube nos tempos de pós-verdade: Educação em saúde ou desinformação? Revista Mídia E Cotidiano, 14(1), 220-239. https://doi.org/10.22409/rmc.v14i1.38210

Ditekemena, J. D., Nkamba, D. M., Mutwadi, A., Mavoko, H. M., Fodjo, J. N. S., Luhata, C., Obimpeh, M., Van Hees, S., Nachega, J. B., \& Colebunders, R. (2021). Covid-19 vaccine acceptance in the democratic republic of congo: A cross-sectional survey. Vaccines, 9(2), 1-11. Scopus. https://doi.org/10.3390/vaccines9020153

Doherty, I. A., Pilkington, W., Brown, L., Billings, V., Hoffler, U., Paulin, L., Kimbro, K. S., Baker, B., Zhang, T., Locklear, T., Robinson, S., \& Kumar, D. (2021). COVID-19 vaccine hesitancy in underserved communities of North Carolina. PLoS ONE, 16(11), e0248542. https://link.gale.com/apps/doc/A680918570/AONE?u=capes\&sid=bookmark-AONE\&xid=fa476aea

Dror, A. A., Eisenbach, N., Taiber, S., Morozov, N. G., Mizrachi, M., Zigron, A., Srouji, S., \& Sela, E. (2020). Vaccine hesitancy: The next challenge in the fight against COVID-19. European Journal of Epidemiology, 35(8), 775-779. Scopus. https://doi.org/10.1007/s10654-020-00671-y

Dula, J., Mulhanga, A., Nhanombe, A., Cumbi, L., Júnior, A., Gwatsvaira, J., Siewe Fodjo, J. N., De Moura Villela, E. F., Chicumbe, S., \& Colebunders, R. (2021). Covid-19 vaccine acceptability and its determinants in mozambique: An online survey. Vaccines, 9(8), Article 8. Scopus. https://doi.org/10.3390/vaccines 9080828

Edwards, B., Biddle, N., Gray, M., \& Sollis, K. (2021). COVID-19 vaccine hesitancy and resistance: Correlates in a nationally representative longitudinal survey of the Australian population. PLoS ONE, 16(3 March), Article 3 March. Scopus. https://doi.org/10.1371/journal.pone.0248892

El-Elimat, T., AbuAlSamen, M. M., Almomani, B. A., Al-Sawalha, N. A., \& Alali, F. Q. (2021). Acceptance and attitudes toward COVID-19 vaccines: A crosssectional study from Jordan. PLoS ONE, 16(4 April), Article 4 April. Scopus. https://doi.org/10.1371/journal.pone.0250555

Fineout-Overholt, E., Melnyk, B.M., Stillwell, S.B., \& Williamson, K.M. (2010). Critical Appraisal of the Evidence: Part I. An introduction to gathering, evaluating, and recording the evidence. AJN, $110(7), 47-52$.

Gatwood, J., McKnight, M., Fiscus, M., Hohmeier, K. C., \& Chisholm-Burns, M. (2021). Factors influencing likelihood of COVID-19 vaccination: A survey of Tennessee adults. American journal of health-system pharmacy: AJHP: official journal of the American Society of Health-System Pharmacists, 78(10), 879889. Scopus. https://doi.org/10.1093/ajhp/zxab099

Harapan, H., Wagner, A. L., Yufika, A., Winardi, W., Anwar, S., Gan, A. K., Setiawan, A. M., Rajamoorthy, Y., Sofyan, H., \& Mudatsir, M. (2020). Acceptance of a COVID-19 Vaccine in Southeast Asia: A Cross-Sectional Study in Indonesia. Frontiers in Public Health, 8, 381. https://doi.org/10.3389/fpubh.2020.00381

Jimenez-Corona, M. E., Aguilar-Díaz, F. D. C., Leon-Solis, L. E., Morales-Virgen, J. J., \& de Leon-Rosales, S. P. (2012). Knowledge, attitudes and practices about influenza A(H1N1) 2009, and influenza vaccine in Mexico: Results of a population survey. Salud Publica de Mexico, 54(6), 607-615. Scopus. https://doi.org/10.1590/S0036-36342012000600009

Khubchandani, J., Sharma, S., Price, J. H., Wiblishauser, M. J., Sharma, M., \& Webb, F. J. (2021). COVID-19 Vaccination Hesitancy in the United States: A Rapid National Assessment. Journal of Community Health, 46(2), 270-277. Scopus. https://doi.org/10.1007/s10900-020-00958-x

Kreps, S., Prasad, S., Brownstein, J. S., Hswen, Y., Garibaldi, B. T., Zhang, B., \& Kriner, D. L. (2020). Factors Associated With US Adults' Likelihood of Accepting COVID-19 Vaccination. JAMA network open, 3(10), e2025594. Scopus. https://doi.org/10.1001/jamanetworkopen.2020.25594

Lazarus, J. V., Ratzan, S. C., Palayew, A., Gostin, L. O., Larson, H. J., Rabin, K., Kimball, S., \& El-Mohandes, A. (2021). A global survey of potential acceptance of a COVID-19 vaccine. Nature Medicine, 27(2), 225-228. Scopus. https://doi.org/10.1038/s41591-020-1124-9

Lin, Y., Hu, Z., Zhao, Q., Alias, H., Danaee, M., \& Wong, L. P. (2020). Understanding COVID-19 vaccine demand and hesitancy: A nationwide online survey in China. PLoS neglected tropical diseases, 14(12), e0008961. Scopus. https://doi.org/10.1371/journal.pntd.0008961

MacDonald, N.E., SAGE, Working Group on Vaccine Hesitancy. (2015). Vaccine hesitancy: definition, scope and determinants. Vaccine, 33(34), 4161-4. https://doi.org/10.1016/j.vaccine.2015.04.036

Malik, A. A., McFadden, S. M., Elharake, J., \& Omer, S. B. (2020). Determinants of COVID-19 vaccine acceptance in the US. EClinicalMedicine, 26. Scopus. https://doi.org/10.1016/j.eclinm.2020.100495

Martins KM,Santos WL, ÁlvaresACM. A importância da imunização: revisão integrativa. Rev Inic Cient Ext. 2019; 2(2): 96-101. Disponível em: https://revistasfacesa.senaaires.com.br/index.php/iniciacao-cientifica/article/view/153/108

Meñaca, A., Tagbor, H., Adjei, R., Bart-Plange, C., Collymore, Y., Ba-Nguz, A., Mertes, K., \& Bingham, A. (2014). Factors likely to affect community acceptance of a malaria vaccine in two districts of ghana: A qualitative study. PLoS ONE, 9(10), Article 10. Scopus. https://doi.org/10.1371/journal.pone.0109707

Mendes, K.D.S.; Silveira, R.C.C.P.; Galvão, C.M. Revisão integrativa: método de pesquisa para a Incorporação de evidências na saúde e na enfermagem. Texto Contexto Enferm, v.17, n4, p. 758-64, 2008. Disponível em: https://www.scielo.br/pdf/tce/v17n4/18.pdf.

Nowak, G. J., Cacciatore, M. A., \& Len-Ríos, M. E. (2018). Understanding and increasing influenza vaccination acceptance: Insights from a 2016 national survey of U.S. adults. International Journal of Environmental Research and Public Health, 15(4), Article 4. Scopus. https://doi.org/10.3390/ijerph15040711

Page, M. J., McKenzie, J. E., Bossuyt, P. M., Boutron, I., Hoffmann, T. C., Mulrow, C. D., Shamseer, L., Tetzlaff, J. M., Akl, E. A., Brennan, S. E., Chou, R., Glanville, J., Grimshaw, J. M., Hróbjartsson, A., Lalu, M. M., Li, T., Loder, E. W., Mayo-Wilson, E., McDonald, S., ....Moher, D. (2021). The PRISMA 2020 statement: An updated guideline for reporting systematic reviews. BMJ, 372, n71.

https://doi.org/10.1136/bmj.n71 
Research, Society and Development, v. 11, n. 1, e0911124194, 2022

(CC BY 4.0) | ISSN 2525-3409 | DOI: http://dx.doi.org/10.33448/rsd-v11i1.24194

Quinn, S. C., Parmer, J., Freimuth, V. S., Hilyard, K. M., Musa, D., \& Kim, K. H. (2013). Exploring communication, trust in government, and vaccination intention later in the 2009 H1N1 pandemic: Results of a national survey. Biosecurity and Bioterrorism: Biodefense Strategy, Practice, and Science, 11(2), 96106. https://doi.org/10.1089/bsp.2012.0048

Riccio, M. D., Boccalini, S., Rigon, L., Biamonte, M. A., Albora, G., Giorgetti, D., Bonanni, P., \& Bechini, A. (2021). Factors influencing sars-cov-2 vaccine acceptance and hesitancy in a population-based sample in Italy. Vaccines, 9 (6), Article 6. Scopus. https://doi.org/10.3390/vaccines9060633

Sacramento, Y. (2021). Fatores que interferem nos índices de coberturas vacinais: uma revisão integrativa. Disponível em: http://ri.ucsal.br:8080/jspui/bitstream/prefix/4521/1/TCCYURISACRAMENTO.pdf

Santos, C.M.C.; Pimenta, C.A.M.; Nobre, M.R.C. (2007). Estratégia Pico para a construção da pergunta de pesquisa e busca de evidências. Rev. Latino-am. Enfermagem, v. 15, n. 3. Disponível em: https://www.scielo.br/pdf/rlae/v15n3/pt_v15n3a23.pdf.

Sato, A.P.S. (2015). What is the importance of vaccine hesitancy in the drop of vaccination coverage in Brazil?. Revista de Saúde Pública [online], 52 (96). https://doi.org/10.11606/S1518-8787.2018052001199.

Silva, L.E.L., Oliveira, M.L.C., \& Galato, D. (2019). Receptividade à vacina contra o papilomavírus humano: uma revisão sistemática. Rev Panam Salud Publica, 43, e22. https://doi.org/10.26633/RPSP.2019.22

Souza, M.T.; Silva, M.D.; Carvalho, R. Revisão integrativa: o que é e como fazer. Einstein. v. 8, n.1, p. 102-6, 2010. Disponível em: https://doi.org/10.1590/s1679-45082010rw1134.

Urrunaga-Pastor, D., Bendezu-Quispe, G., Herrera-Añazco, P., Uyen-Cateriano, A., Toro-Huamanchumo, C. J., Rodriguez-Morales, A. J., Hernandez, A. V., \& Benites-Zapata, V. A. (2021). Cross-sectional analysis of COVID-19 vaccine intention, perceptions and hesitancy across Latin America and the Caribbean. Travel Medicine and Infectious Disease, 41. Scopus. https://doi.org/10.1016/j.tmaid.2021.102059 